\title{
Nonlinear association between CGG repeat number and age of menopause in FMR1 premutation carriers
}

\author{
Sarah Ennis ${ }^{1}$, Daniel Ward ${ }^{2}$ and Anna Murray*,2 \\ ${ }^{1}$ Genetic Epidemiology \& Bioinformatics Group, Human Genetics Division, University of Southampton, Southampton, \\ UK; ${ }^{2}$ Wessex Regional Genetics Laboratory, Salisbury District Hospital, Salisbury, UK
}

FMR1 premutations are known to be associated with premature ovarian failure (POF), but the underlying mechanism is unknown. We present evidence for a nonlinear association between menopause age and premutation size suggesting that premutations in the mid-size range are at greatest risk for POF, while larger premutations are at lower risk.

European Journal of Human Genetics (2006) 14, 253-255. doi:10.1038/sj.ejhg.5201510; published online 26 October 2005

Keywords: FMR1; permutation; premature ovarian failure

\section{Introduction}

Expansions of the CGG repeat in FMR1 over about 200 copies are associated with methylation and subsequent gene inactivation, which causes the fragile $\mathrm{X}$ syndrome. Full mutations arise through maternal expansion from a premutation. Premutations have 50-200 CGGs and are unmethylated. Three phenotypes have been associated with FMR1 premutations: (1) learning difficulty; ${ }^{1}$ (2) premature ovarian failure ( $\mathrm{POF})^{2,3}$ and (3) fragile-X-associated tremor/ataxia syndrome (FXTAS). ${ }^{4,5}$ The underlying molecular mechanism is unknown, but full mutation females are not affected with POF, suggesting that reduced FMR1 protein levels do not affect ovarian function. ${ }^{3}$ There is a significant increase in FMR1 mRNA with increasing repeat number, leading to speculation of an RNA mediated gain-of-function mechanism, similar to that seen in myotonic dystrophy. ${ }^{6}$ If FMR1 expanded RNA is pathogenic, one might expect to see anticipation in affected families, such that as repeat number increased, phenotype became more severe or had an earlier age of onset. We have investigated the affect of CGG repeat number on menopause age.

*Correspondence: Dr A Murray, Peninsula Medical School, St Lukes, Magdalen Road, Exeter, EX1 2LU, UK. Tel: + 441392 262925;

Fax: + 441722 338095; E-mail: anna.murray@pms.ac.uk

Received 25 May 2005; revised 2 September 2005; accepted 16 September 2005; published online 26 October 2005
Materials and methods

We tested 45 postmenopausal premutation carriers from 32 families, ascertained through a retarded proband with no knowledge of menopause history. All individuals had ceased menstruating for at least 6 months without known cause. Menstrual histories were obtained by interview, and data on a proportion of this cohort have been published previously. ${ }^{7}$ In the original study many premutations were not detected by PCR and an estimate of size was made from conventional Southern blots. We have reanalysed all premutations by two methods, on consecutive gels. Genomic DNA (100 ng) was amplified with primers c and $\mathrm{f}^{8}$ using the GC-rich PCR system (Roche Diagnostics, UK). Products were separated on $2 \%$ agarose gels and sizes were measured by comparison with 100 bp ladder using Syngene software. Ten $\mu$ l of PCR product was separated by denaturing PAGE and transferred to nylon membrane. Blots were hybridized with a $\mathrm{CGG}_{5}$ digoxygenin (DIG) labeled oligonucleotide and visualized with chemiluminescent detection (Roche Diagnostics, UK). Bands were sized relative to a DIG labeled size marker.

The agarose gel method gave the most consistent and accurate measure of size for $41 / 45$ samples. The remaining four were only detected following Southern blot and hybridization to the PCR product. A comparison of the two methods showed a significant difference between allele sizing ( $t=2.982, P=0.005$ ). Further investigation of the relationship between allele measurements from the 
two methods using regression analysis, showed the relationship was principally linear $(P<0.0001)$ with a smaller but significant quadratic effect $(P=0.012)$. The Southern blot method underestimated allele length in smaller repeats and overestimated it in the larger repeats. For the four cases with only a Southern blot result, parameter estimates from this regression were used to predict the direct method allele size. These four predicted values plus the 41 successful direct PCR results were used to determine the relationship between repeat size and menopause age.

\section{Results}

Regression analysis showed a significant relationship between premutation size and menopause age using a quadratic model (Figure $1, R^{2}=0.22, \quad F=5.9197$, $P=0.0054, d f=2,42$ ) with a minimal value at 80 repeats. There was no evidence for a linear effect $\left(R^{2}=0.01\right.$, $F=0.3978, P=0.5316, d f=1,43)$. Three women had menopause ages less than 30 years and could possibly be construed as outliers. These women were removed from the analysis, the quadratic model retested and the relationship remained significant $\left(R^{2}=0.18, F=4.2476, P=0.0214\right.$, $d f=2,39$ ). However, given that these women are not related and that they are unlikely to have made a significant error in their reported age of menopause given the acute consequences on their reproductive lives, we believe their data to be accurate and their inclusion in the model justified. The nonlinear association of menopause age with premutation size indicates that while premutations with less than 80 repeats demonstrate increasing severity of the phenotype with increasing repeat number, over 80 repeats there is a reduction in phenotype severity with increasing repeat number. We investigated this relationship further by dividing the data into those with

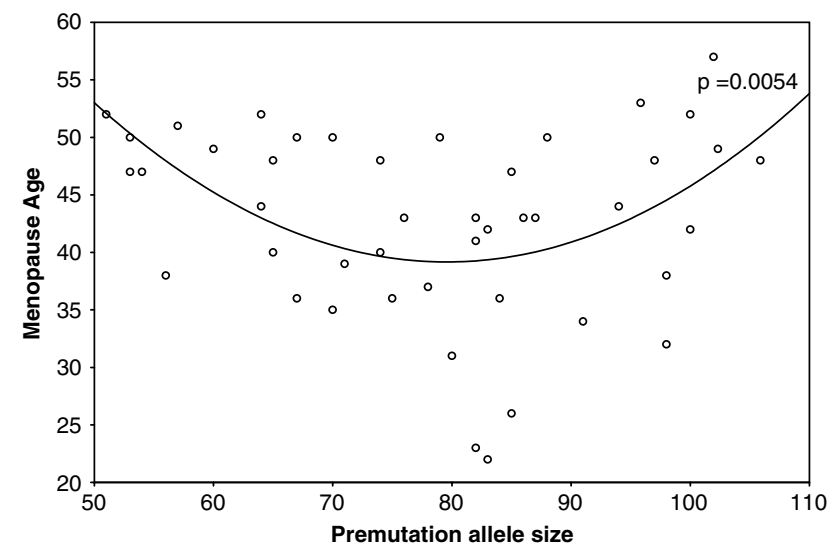

Figure 1 Relationship between menopause age and CGG repeat size.
FRAXA alleles $<80$ repeats and those with FRAXA alleles $>80$ repeats and examined the linear effects. In agreement with Sullivan et $a l^{9}$ there is suggestive evidence of a linear decline in menopause age with increasing repeat size in those individuals with alleles in the range of 50-80 repeats $\left(R^{2}=0.16, F=3.9519, P=0.0607, d f=1,20\right)$, lack of formal significance is presumably a function of diminished sample size and power when the data are split $(n=22)$. A significant positive linear association between repeat size and age of menopause is seen in the women with premutations in the $80-110$ repeat range $\left(R^{2}=0.31\right.$, $F=9.2686, P=0.0062, d f=1,21)$ despite the small sample size $(n=23)$. This linearity remained statistically significant $(P=0.0494)$ even when the three women with very low menopause age were removed from the analysis.

\section{Discussion}

Preliminary evidence for a nonlinear association was presented by our group in 2004 (Murray et al, pers comm). Sullivan et $a l^{9}$ also reported a positive association between repeat size and ovarian dysfunction in smaller premutations and while the data were suggestive of a plateau over 100 repeats they were not significant. We have used our method to determine the size of five premutations included in Sullivan et $a l^{9}$ and they were all within five repeats of the size calculated in the Atlanta study, making the two studies comparable with respect to sizing methods. Sullivan et al also suggested that carriers with $>100$ repeats may go through menopause around the same age as noncarriers, but analysis was limited by the relatively small sample size. We also had few women with $>100$ repeats and intend to collaborate with other groups to increase the number of individuals in this category.

Our data demonstrate a statistically significant nonlinear association between menopause age and premutation size, such that menopause age decreases with increasing repeat number until about 80 repeats, thereafter increasing repeat number is associated with increasing menopause age. There are several hypotheses to explain our observations:

1. As premutation carriers are at risk of transmitting full mutations to their offspring, a proportion of oocytes in premutation carriers may in fact have full mutations, with the proportion increasing with increasing premutation size. Full mutations are not at increased risk of POF, therefore females with large premutations in their peripheral blood may be more like full mutations with respect to ovarian failure.

2. In an RNA-mediated model, it is likely that secondary structure is an important factor and perhaps CGG repeats in the mid-premutation range adopt a particularly deleterious structure. This may allow inappropriate protein binding or affect localisation of the transcript within cells. 
3. Recent studies have demonstrated the presence of three alternative transcription start sites for FMR1 and the relative proportion of these varies with repeat number. ${ }^{10}$ It is possible that these qualitative differences in RNA transcripts play a role in the aetiology of premutation phenotypes.

4. The positive association between menopause age and CGG repeat numbers for premutations $>80$ could be a result of ascertainment bias. Mothers and grandmothers of fragile X males have, by definition, had at least some reproductive success and may have larger FRAXA alleles because the premutation has expanded to a full mutation. POF often results in infertility, thus mothers and grandmothers of fragile $\mathrm{X}$ probands could have a low risk of POF. We interrogated our data and found no evidence that mothers or maternal grandmothers were associated with larger premutations $(P=0.9)$.

These hypotheses are not mutually exclusive and may act in combination.

In summary, we have found that increasing repeat size is associated with increased risk of ovarian failure for premutations under 80 repeats, but larger premutations are in fact at reduced risk for POF. The data support an RNA-mediated gain-of-function mechanism, but disease severity may not simply be a function of RNA quantity. The presence of full mutation oocytes in the ovary may also explain our nonlinear association data: there may be a trade-off between increasing mRNA as repeat number increases and decreasing mRNA as large premutations expand to full mutations in oocytes.

\section{Acknowledgements}

We thank Professors Pat Jacobs and Newton Morton for their support and advice. This work was funded by the Wellcome Trust.

\section{References}

1 Youings SA, Murray A, Dennis $\mathrm{N}$ et al: FRAXA and RAXE: the results of a five year survey. J Med Genet 2000; 37: 415-421.

2 Murray A, Webb J, Grimley S, Conway G, Jacobs P: Studies of FRAXA and FRAXE in women with premature ovarian failure. J Med Genet 1998; 35: 637-641.

3 Allingham-Hawkins DJ, Babul-Hirji R, Chitayat D et al: Fragile X premutation is a significant risk factor for premature ovarian failure: the International Collaborative POF in Fragile X studypreliminary data. Am J Med Genet 1999; 83: 322-325.

4 Hagerman RJ, Leavitt BR, Farzin F et al: Fragile-X-associated tremor/ataxia syndrome (FXTAS) in females with the FMR1 premutation. Am J Hum Genet 2004; 74: 1051-1056.

5 Hagerman RJ, Leehey $\mathrm{M}$, Heinrichs $\mathrm{W}$ et al: Intention tremor, parkinsonism, and generalized brain atrophy in male carriers of fragile X. Neurology 2001; 57: 127-130.

6 Tassone F, Hagerman RJ, Taylor AK, Gane LW, Godfrey TE, Hagerman PJ: Elevated levels of FMR1 mRNA in carrier males: a new mechanism of involvement in the fragile-X syndrome. Am J Hum Genet 2000; 66: 6-16.

7 Murray A, Ennis S, MacSwiney F, Webb J, Morton NE: Reproductive and menstrual history of females with fragile $\mathrm{X}$ expansions. Eur J Hum Genet 2000; 8: 247-252.

$8 \mathrm{Fu}$ YH, Kuhl DPA, Pizzuti A et al: Variation of the CGG Repeat at the Fragile-X Site Results in Genetic Instability - Resolution of the Sherman Paradox. Cell 1991; 67: 1047-1058.

9 Sullivan AK, Marcus M, Epstein MP et al: Association of FMR1 repeat size with ovarian dysfunction. Hum Reprod 2005; 20: $402-412$.

10 Beilina A, Tassone F, Schwartz PH, Sahota P, Hagerman PJ: Redistribution of transcription start sites within the FMR1 promoter region with expansion of the downstream CGG-repeat element. Hum Mol Genet 2004; 13: 543-549. 